\title{
Optimizing the focal laws of ultrasonic arrays for non-destructive evaluation of composite panels with delamination
}

\author{
Mariam Achbal ${ }^{1}{ }^{1, a}$, Abdellatif Khamlichi ${ }^{2}$ and Fadoua El Khannoussi ${ }^{2}$ \\ ${ }^{1}$ Department of Physics, FS Tetouan, 93000 Tetouan, Morocco \\ ${ }^{2}$ Department of Industrial and Civil Sciences and Technologies, ENSA Tetouan, 93000 Tetouan, Morocco
}

\begin{abstract}
The use of ultrasonic arrays in the field of non-destructive evaluation of composites is a recent technique. This method offers the possibility to achieve fast inspection of components having complex geometries. As compared with the single element transducers, arrays configuration yields more effective detection of defects. However, to achieve adequate imaging by ultrasonic array elements, each element should be excited according to a specific sequence which is called the focal law. At each instant a subset of the array elements is activated and the incident waves are better directed to scattered at the defect. The reflected signals can then be more intensified. In this work, two-dimensional approximation of wave propagation problem was supposed. Array elements with linear geometry and with the elements assumed to be fully coupled with the composite panel were considered. A delamination defect was inserted between the plies of the composite panel at a given depth. The forward model enabling to simulate ultrasonic array data was built by means of the FEM. As each focal position requires a different focal law that fixes a proper protocol of excitation and reception, synthesis of these focal laws was studied. Optimization of signal focalization and reception as function of the delamination extent was achieved.
\end{abstract}

\section{Introduction}

Composites are made from various combinations of the three basic categories of materials as metals, polymers and ceramics to form the fourth basic category of materials. They consist of two or more separate materials combined in a structural unit. Composites are normally used because they have the required properties for a giving application that cannot be reached by any of the constituent materials acting alone. Because composites are the combination of two visibly distinct constituents into a single material, fibers and matrix, they present a large variety of damages that could be specific to one or both of the constituents or involve interaction of the two. the failures modes that often face the composites structures are matrix cracks, fiber/matrix interface failures, fiber failures, through-thickness failures (holes and cracks) and inter-ply failures such as delamination $[1,2]$. The composites properties allowed them to be used in a wide variety of industries, including aerospace that has demands for high-quality, cost-effective solutions in design, fabrication, and inspection. Since the aerospace structures are very sensitive to damage which can has various sources, regular inspection of the composite structures is necessary. Because of their anisotropy, the inspection of composites is not an easy task. Many of the inspection problems posed by composites can be dealt with effectively using ultrasonics $[3,4]$. Phased array ultrasonic testing (PAUT) is one of the advanced inspection technologies that promises to become the primary inspection technique for a large number of composites applications $[5,6]$. An array probe consists of a multiple transducer elements, which can be excited independently with appropriate time delays. The combination of sound waves generated by the transducer elements forms an intense sound beam. This sound beam propagates through a component and may reveal the presence of defects by reflecting the beam scattered on the flaws to a transducer. The time of exciting individual transducer elements is phased in order to direct and shape the sound beam. The variation of the pulsing timing changes condition how the sound waves combine with each other constructively or destructively.

Due to its inspection flexibility and ability to increase the inspection quality compared to the single element transducer, phased array (PA) transducers technology represents such an effective method of inspecting composite structures. This emphasizes the main motivation of this work aiming at improving the advantages that the PAUT to overcome the difficulties of inspecting composite structures.

Modeling and simulation are important tools to determine the optimal focal laws that enable to optimize the beamforming properties of the array and to evaluate the sound wave propagation through the component. The literature is rich of works about simulation models to determine the sound beam produced by PA probes. Buiochi et al. [7] developed a 3D computational method

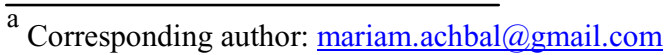


based on the spatial impulse response and on the discrete representation concept to simulate acoustic beams generated by arrays through arbitrary shape of interfaces. They used this modeling method to optimize the beam focusing as produced by arrays and propagated through interfaces of complex geometry. This was performed by calculating the optimal focal law. Additionally, these authors compared two different focusing techniques and found that the cross-correlation technique could give more precise focalizing than the ray approximation technique.

The focal laws are one of the parameters that affect the beamforming. In the commercial PA probes that exist nowadays, the focal laws generators are based on the geometrical approach where the only parameter considered is the pitch between the single elements. The elements are approximated by a point source in the center of their aperture. Furthermore, the wave propagation process itself is modelled by geometrical acoustics. The traditional formulas to calculate the focusing delays are not affected by the width or length of the single transducer elements. The only geometrical value which influences the focal laws is the distance between the centers of adjoined elements (the pitch) [8]. In this context, Gommlich and Schubert [8] had created different simulation aperture models to study the influence of aperture active and passive size on the resulting signals in the focal point. The results had shown that the real aperture size, in particular the active size, has an important impact on the signals. These results had led to calculate improved focal laws by considering the real aperture size and the full wave phenomena in the phased array near-field.

In another work, Dheeraj et al. [9] discussed the effect of different focal law parameters including element quantity, pitch, focal depth, range of angle, and angle resolution, in order to insure an effective inspection. To do so, they analyzed C-scan plans using simulation and practical trials to derive the best scan plan in terms of detectability, resolution and sizing of defects. The study concludes that for an effective PAUT examination, focal law parameters must be validated either through practical or simulation approach. A simulation to evaluate the smallest detectable discontinuity based on effective scan plan is proposed.

The scope of this work is to optimize the focal law of an ultrasonic linear array probe in order to maximize delamination defect detection in composites. The PA ultrasonic modeling was performed by using ABAQUS Software. Different models of a composite panel made of laminated Carbon Fiber Reinforced Epoxy (CFRE) layers and containing a delamination defect at different depths were numerically analyzed. An optimization of the pitches to maximize focalization and reception of the beam will be conducted. The influence of the position of the receiver and the delamination depth will be studied.

\section{Materials and methods}

In the simplest cases, the elastic wave equation could have an analytical solution. But, to model wave propagation for more complicated structures and at high frequencies such as in ultrasonic waves, the analysis becomes more difficult. This is why approximate solutions as provided by numerical solutions should be searched for in that case. In structural mechanics and acoustics fields, use is often made of the finite element method as a basic modeling approach.

In this paper, modeling was carried out based on the finite element method by using ABAQUS software. The ultrasonic array probe parameters, the shape and the size of the inspected part and the materiel of the sample were defined as input data. Plain strain hypothesis was assumed and adequate boundary conditions were applied. The duration of calculation was scaled in order to avoid reflections from the boundaries.

\subsection{Sample and Phased Array modelling}

A 2-D panel simulation model using ABAQUS is created. This has the form of a composite plate made of seven bonded plies of CFRE laminates. A delamination defect was inserted between the plies of the panel. The flaw was modelled as a vacuum separating the materiel plies. Description of the modeled panel geometry, material characteristics, size depth and type of the defect in the sample are presented in tables 1 and 2.

Table 1. Materiel properties and panel dimensions

\begin{tabular}{|c|c|c|c|}
\hline Materiel & $\begin{array}{c}\text { Plies } \\
\text { number }\end{array}$ & $\begin{array}{c}\text { Dimensions } \\
\mathrm{mm}^{2}\end{array}$ & $\begin{array}{c}\text { Structure } \\
\text { Lay-up }\end{array}$ \\
\hline $\begin{array}{c}\text { Carbon Fibre } \\
\text { Reinforced Epoxy } \\
\text { (CFRE) Laminate }\end{array}$ & 7 & $157.5 \times 0.875$ & $\begin{array}{c}{\left[0^{\circ}, 90^{\circ}, 0^{\circ}\right.} \\
, 90^{\circ}, 0^{\circ} \\
\left., 90^{\circ}, 0^{\circ}\right]\end{array}$ \\
\hline
\end{tabular}

Table 2. Defect characteristics

\begin{tabular}{|l|c|}
\hline Defect & delamination \\
\hline $\begin{array}{l}\text { Defect size } \\
(\mathrm{mm})\end{array}$ & $17.5 \times 0.4$ \\
\hline $\begin{array}{c}\text { Location/ } \\
\text { Depth (mm) }\end{array}$ & 4th ply / 0.42 \\
\hline
\end{tabular}

In this study a linear array probe of 16 elements was modeled, see figure 1 . The elements are assumed to be fully coupled with the composite panel. The excitation time signal $g(t)$ of each element of the probe has the form of an oscillating pulse centered on the work frequency. It form is given by equation 1 .

$$
g(t)=\mathrm{e}^{-\mathrm{at} t^{2}} \sin (2 \pi \mathrm{f} t)
$$

where $\mathrm{f}=1.5 \mathrm{MHz}$ is the central frequency, $\mathrm{t}$ the time and a the amplitude parameter.

The length of each element is $1.75 \mathrm{~mm}$ and the total length of PA patch is $28 \mathrm{~mm}$. 


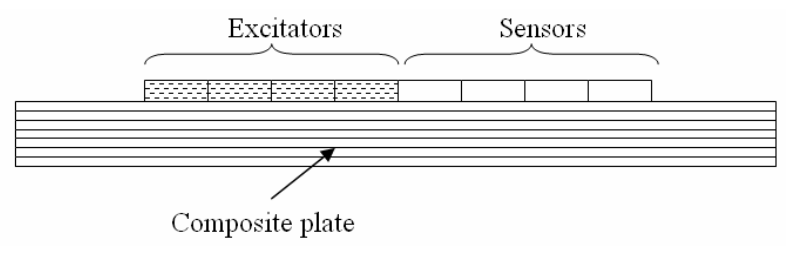

Figure 1. Testing configuration using a linear PAUT of 16 elements.

\subsection{Excitation and Reception Protocol}

One of the main advantages of the PA based inspection technology is its ability to perform more effectively inspection. In this work where a PA consisting of 16 linear elements is considered, the configuration of the inspection probe shown in figure is assumed to use the first 8 elements as excitators, while the response of the system is measured by the last 8 elements which play the role of sensors. Other configurations are possible by changing the number and location of excitators and sensors. In the following, only the previous pattern made of 8 excitators and 8 sensors is investigated.

To detect the presence of delamination, an indicator signal is defined as the difference between the system response in the model where the delamination defect was inserted and that one provided by a defect free model.

\subsection{Additional models}

To assess the effect of delamination depth two additional models with a delamination defect inserted between their plies were considered. The delamination size was kept fixed and the delamination depth is indicated in table 3.

Table 3. Defect characteristics in the additional models

\begin{tabular}{|c|c|c|}
\hline Defect & Delamination & Delamination \\
\hline $\begin{array}{c}\text { Defect size } \\
(\mathrm{mm})\end{array}$ & $17.5 \times 0.4$ & $17.5 \times 0.4$ \\
\hline $\begin{array}{c}\text { Location/ } \\
\text { Depth }(\mathrm{mm})\end{array}$ & 2nd ply / 0.42 & 6th ply / 0.42 \\
\hline
\end{tabular}

\section{Results and discussion}

\subsection{First modelling results}

The PA probe and host medium systems are assumed to be linear, so they comply with the principal of superposition. The actual system's excitation input can be regarded to be the sum of each individual element excitation input function. Also, for the reason of the system's linearity, the response of the system can be calculated as the sum of individual responses of each element.

Considering the probe configuration mentioned above, the envelopes of individual responses of the system are plotted in figure 2. These correspond to the individual responses measured for each single sensor taken separately. The response consists of the reflected signal by the defect following the excitation initiated by a single element. It can be noticed that the intensity of received signals depends on the location of the excitator element. The signal intensity can be improved by adopting a PA inspection strategy through optimizing the focal laws that control the focalization and the reception of the signal.

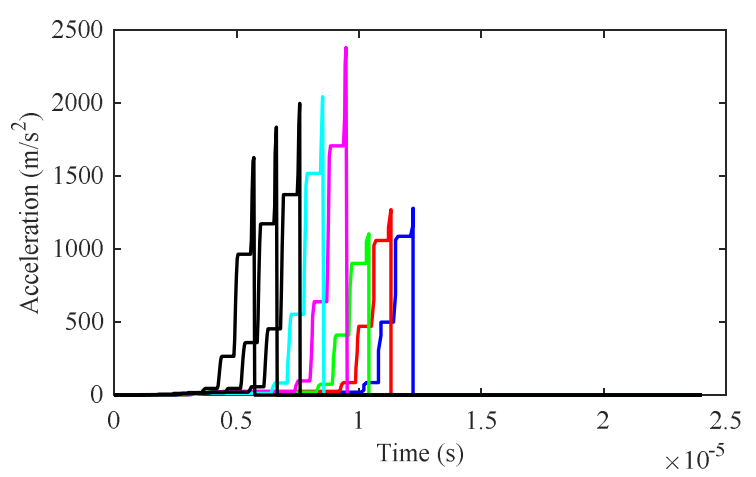

Figure 2. The envelopes of the individual responses at sensor 4 of the PA system.

\subsection{Optimization results}

In order to maximize the intensity of the received signal, an optimization algorithm using MATLAB was developed. The main objective of this algorithm is to calculate the optimized focal laws, and this was performed by computing the excitation phases required to receive the maximal amplitude of each individual response at the same instant.

The calculation of the optimized focal laws was achieved for each one of the eight reception sensors. The resulted signals after optimizing showed that the signal which presents the defect echo is much more intense than that associated to a single element excitation, see figure 3 .

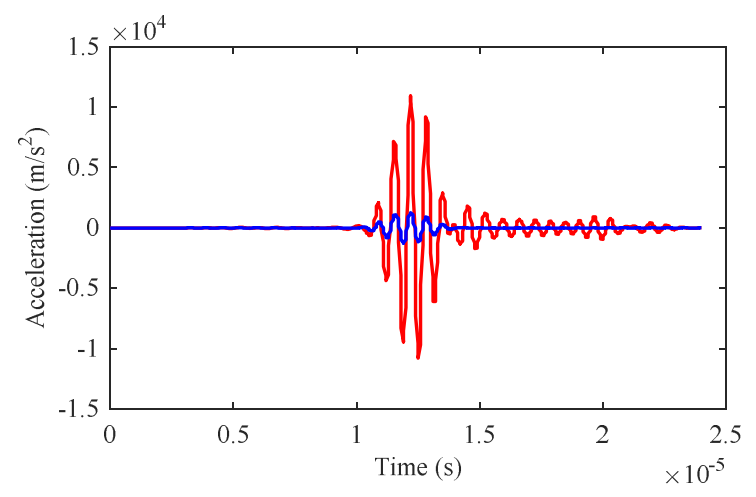

Figure 3. Received signal at sensor 4: a single exicator (blue); Phased array excitation (Red)

\subsection{Effect of sensor position and defect depth}

By comparing the signals received in each sensor, it could be concluded that the position of the sensor influences both the time of receiving the signal and the signal amplitude. More the position of the sensor is far 
from the excitation area, more the received signal amplitude is small. This is due to geometric dispersion of waves. This remark justifies why the inspection protocol takes the form of a scan as this enables the PA elements to be enough closer to the defect area.

To study the influence of the defect depth on the system's response, three different positions of the delamination defect were considered.

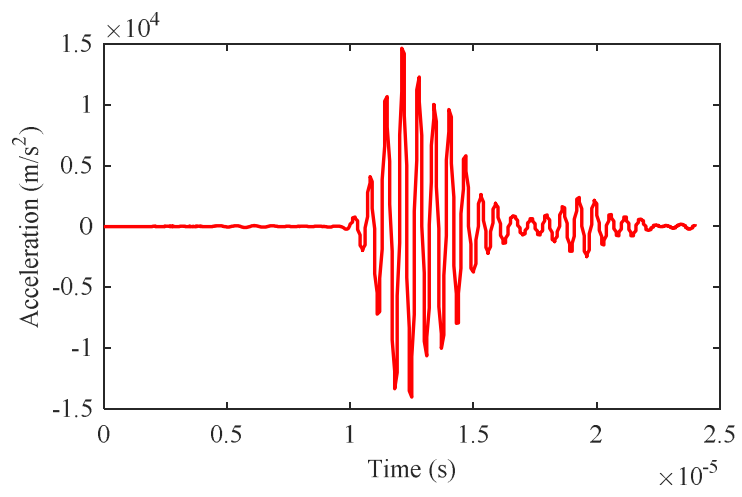

Figure 4. Received signal at sensor 4: Phased array excitation (defect in the $2^{\text {nd }}$ ply)

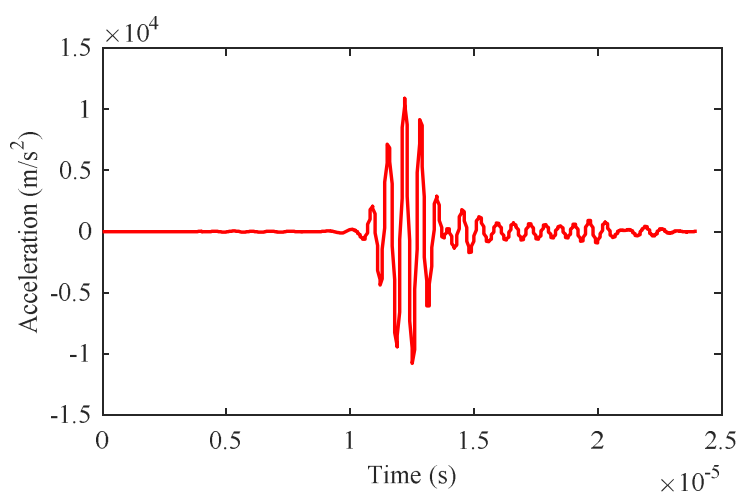

Figure 5. Received signal at sensor 4: Phased array excitation (defect in the $4^{\text {th }}$ ply)

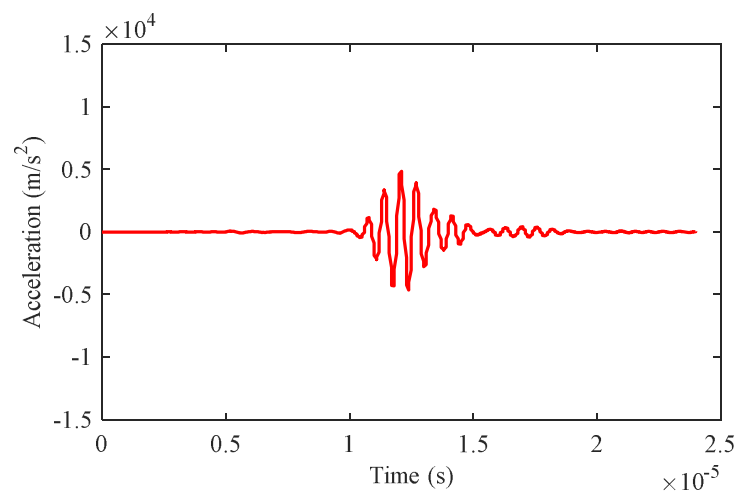

Figure 6. Received signal at sensor 4: Phased array excitation (defect in the $6^{\text {th }}$ ply)

Figures 4,5,6 present successively the optimized received signals in the case where the delamination defect is in the $2^{\text {nd }}$ ply, in the $4^{\text {th }}$ ply and in the $6^{\text {th }}$ ply of the composite panel. It can be seen that the delamination defect depth influences largely the intensity of the received signal and that the amplitude of the echo signal decreases rapidly with defect depth.

\section{Conclusions}

In this work, a 2-D approximation of wave propagation taking place in a composite plate was assumed in order to study detection of delamination by phased array ultrasound probes. Different models were created for the purpose of simulating this linear system. These contained a delamination defect inserted between the plies of the composite panel. A numerical solution of the problem was found using the finite element method.

The numerical simulation results obtained from the PA modelling showed that a linear array probe consisting of 16 elements with a central frequency at $1.5 \mathrm{MHz}$ could provide enhanced inspection of the composite panel. This could be achieved by optimizing the focal laws which control the focalization of the emitted signal and augment the amplitude of the received echo generated by reflection occurring at a defect.

The influence of the sensor position on the reflected signal received was analyzed, as well as the influence of the delamination defect depth. The results of simulations showed that the focal laws enable to intensify the echo by performing delayed firing of the excitators.

As the calculation time for the finite element simulation is rather large, it is recommended to apply in future work another numerical method in order to simulate the PA inspection process. It seems that the scaled boundary finite element method can advantageously be used for simulation of wave propagation in composites suffering from delamination.

\section{References}

1. R. F. Gibson, Principles of composite materiel mechanics (Taylor \& Francis Group, Boca Raton, 2012)

2. C. Kassapoglou, Modelling the effect of damage in composite structures (John Wiley \& Sons Ltd, Hoboken, 2015)

3. C. Fan, M. Caleap, M. Pan, B.W. Drinkwater, Ultrasonics, 54, 1842 (2014)

4. A. Velichko, P.D. Wilcox, J. Acoust. Soc. Am. 127, 2377 (2010)

5. B. Drinkwater, P. Wilcox, NDT \& Int. 39, 525 (2006)

6. O. Martínez, M. Akhnak, L.G. Ullate, F. Montero de Espinosa, NDT \& Int., 36, 57 (2003)

7. F. Buiochi, O. Martinez, L. G. Ullate, F. Montero de Espinosa, Ultrasonics 42, 871 (2004)

8. A. Gommlich, F. Schubert. On Determination of Focal Laws for Linear Phased Array Probes as to the Active and Passive Element Size, $19^{\text {th }}$ World Conference on Non-Destructive Testing, (2016)

9. P.R. Dheeraj, I. Mohsin, S. K. Mohiuddin, S. H. Masroor, M.E. 74, 1574 (2016) 